\title{
Gestión de proyectos aplicado a un juego de Logística
}

\section{Pablo Aparicio-Ruiz}

Grupo Ingeniería de Organización Escuela Técnica Superior de Ingeniería. Universidad de Sevilla (España) Email: pabloapariciodus.es

ORCID: 0000-0002-9979-4183

\section{José Guadix-Martín}

Grupo Ingeniería de Organización Escuela Técnica Superior de Ingeniería. Universidad de Sevilla (España) Email: guadix@us.es

ORCID: 0000-0002-3221-5095

\section{Elena Barbadilla-Martín}

Grupo Ingeniería de Organización Escuela Técnica Superior de Ingeniería. Universidad de Sevilla (España)

Email: ebarbadilladus.es ORCID: 0000-0002-6825-9970

\section{Alejandro Escudero-Santana}

Grupo Ingeniería de Organización Escuela Técnica Superior de Ingeniería. Universidad de Sevilla (España) Email: alejandroescuderodus.es ORCID: 0000-0002-9490-1402

\section{RESUMEN}

La transmisión de conceptos mediante juegos combina una aplicación práctica de la teoría junto con un incremento potencial del interés y la motivación de los alumnos. Este objetivo en el caso de la transmisión de conceptos de la gestión de proyectos ágil, mediante la metodología Scrum, se consigue mediante un juego aplicado a la logística, que se propone en esta comunicación, en la que se explica la dinámica del juego, y cómo se reflejan todos los conceptos de esta metodología ágil.

Palabras clave: Gestión de proyectos, scrum, ágil, juegos, gamificación.

\section{Project management applied to a Logistics game}

\section{ABSTRACT}

The transmission of concepts through games combines a practical application of the theory together with a potential increase in the interest and motivation of the students. This objective in the case of the transmission of agile project management concepts, through the Scrum methodology, is achieved through a game applied to logistics, which is proposed in this communication, in which the dynamics of the game are explained, and how all the concepts of this agile methodology are reflected.

Key words: Project management, scrum, agile, games, gamification. 


\section{Introducción}

En el mundo real, la gestión de proyectos se caracteriza por ser compleja y existir una alta subjetividad a la hora de tomar decisiones. Tomar decisiones es una habilidad crucial si se quiere alcanzar el éxito, por lo que un juego puede ayudar a incorporar la aplicación de estas habilidades. Por otra parte, los juegos en la docencia deben de reflejar las técnicas aplicadas. Este artículo presenta un juego que permite reflejar todos los aspectos de una de las metodologías ágiles de gestión de proyectos, SCRUM.

La aplicación del juego, permite incorporar habilidades como la comunicación, la cultura del trabajo en equipo, la motivación, la resolución de problemas, etc. Los alumnos de Ingeniería de Organización requieren de una formación de habilidades complementarias de carácter transversal (soft skills), por lo que la aplicación de juegos como metodología de enseñanza puede ser muy positiva (Castán et al., 2019).

En la literatura existen juegos aplicados a esta metodología, en la revisión de (Petri et al., 2019) se observan juegos centrados en diferentes aspectos de la gestión de proyectos, y generalmente a través de preguntas o para simular la planificación, ejecución y control de un proyecto. Existen algunos ejemplos de aplicación de juegos en esta área, por ejemplo, (Fernandes \& Sousa, 2010) presentan PlayScrum, un juego de cartas diseñado para ayudar a alumnos de nivel universitario a aprender el método Scrum. En (Sibona et al., 2018) se presentan algunos ejemplos, como: Scrumia, un simulador de SCRUM con LEGO Bricks, etc. donde los autores destacan que, en los juegos analizados, ninguno de ellos varía las tareas al final de un sprint. En ellos, las actividades se centran en diferentes conceptos y requieren cantidades variables de tiempo para completar la actividad, o se centran en el rol del propietario del producto y en la selección de tareas para desarrollar un sprint, otros se centran en la reunión diaria, o el desarrollo iterativo. Este trabajo se enmarca dentro del Plan de Innovación Docente de la Universidad de Sevilla, el juego se encuadra dentro de una propuesta más amplia de actividades entre las que se encuentran juegos, simulaciones empresariales y conferencias (Escudero-Santana et al., 2019).

En este trabajo se presenta una aplicación integral del método Scrum. La sección 2, presenta de manera sucinta el Juego propuesto para la actividad docente. La sección 3 justifica la aplicación de las metodologías ágiles aplicadas al juego. La sección 4, presenta la aplicación del método Scrum en el juego, y se identifican los elementos de la metodología. Por último, unas breves conclusiones.

\section{Juego aplicado a la logística}

A continuación, se presenta el juego con el cual se ha propuesto a los alumnos gestionar la partida aplicando la metodología SCRUM. Se trata de un juego de temática asociada a la logística, donde dos equipos compiten para conectar ubicaciones en un grafo en el cual se definen posibles rutas. Las rutas están representadas por unidades de trayecto que se deben abarcar. La partida, el proyecto, tiene un límite en el número de unidades de trayectos construibles, la cual determina el final del proyecto.

En la Figura 1 se presenta una simplificación del tablero a modo de ejemplo. En el tablero se puede observar que en el trayecto entre Huelva y Córdoba hay 6 unidades de trayecto que deben de ser construidas con recursos del mismo tipo (en el ejemplo, serían cartas de recurso de color naranja).

En el grafo del tablero también existen recorridos, trayectos entre dos ciudades, duplicados, por ejemplo, ir de Huelva a Sevilla, donde un mismo equipo no puede desarrollar dos veces el mismo recorrido. Los recorridos del juego aportan puntos, valor al proyecto, y son desarrollados mediante la consecución de cartas de recursos obtenidas de una baraja de cartas, siguiendo un conjunto de reglas.

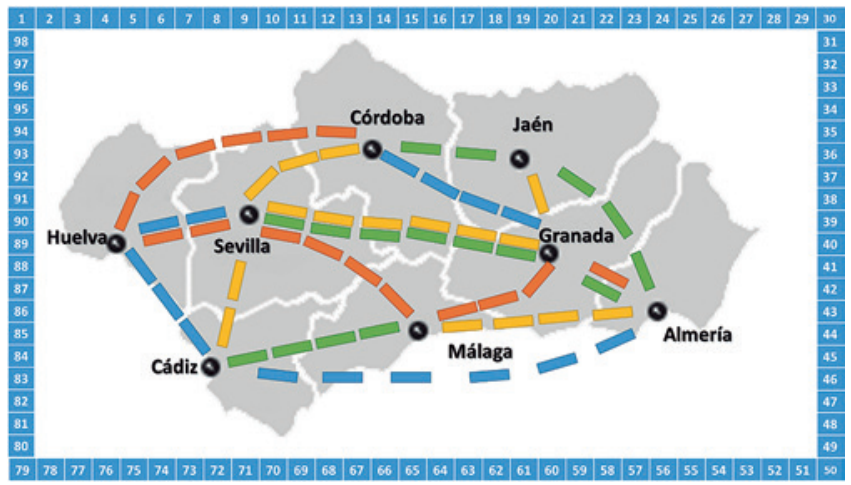

Figura 1. Ejemplo simplificado del tablero de juego.

A continuación, se presenta el juego con el cual se ha propuesto a los alumnos gestionar la partida aplicando la metodología SCRUM. Se trata de un juego de temática asociada a la logística, donde dos equipos compiten para conectar ubicaciones en un grafo en el cual se definen posibles rutas. Las rutas están representadas por unidades de trayecto que se deben abarcar. La partida, el proyecto, tiene un límite en el número de unidades de trayectos construibles, la cual determina el final del proyecto.

En la Figura 1 se presenta una simplificación del tablero a modo de ejemplo. En el tablero se puede observar que en el trayecto entre Huelva y Córdoba hay 6 unidades de trayecto que deben de ser construidas con recursos del mismo tipo (en el ejemplo, serían cartas de recurso de color naranja).

En el grafo del tablero también existen recorridos, trayectos entre dos ciudades, duplicados, por ejemplo, ir de Huelva a Sevilla, donde un mismo equipo no puede desarrollar dos veces el mismo recorrido. Los recorridos del juego aportan puntos, valor al proyecto, y son desarrollados mediante la consecución de cartas de recursos obtenidas de una baraja de cartas, siguiendo un conjunto de reglas.

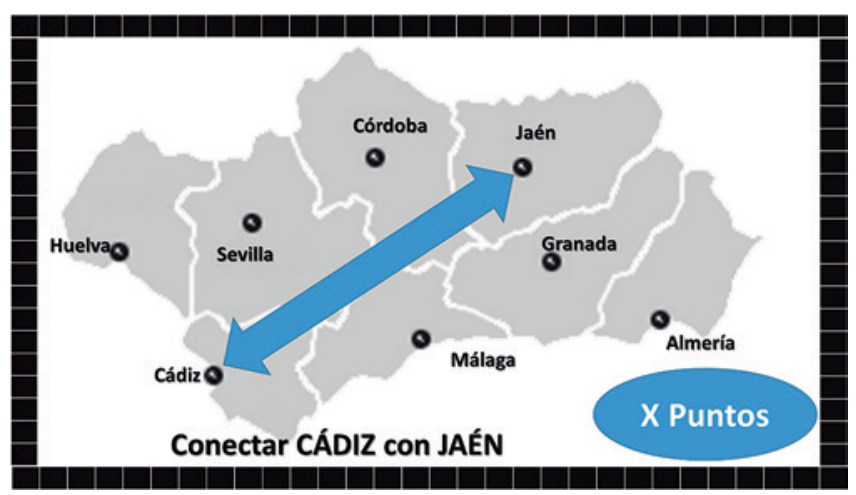

Figura 2. Ejemplo de tarjeta asociada al tablero simplificado del juego.

Por supuesto, el objetivo del juego es alcanzar el mayor número de puntos posibles, si bien, se pierden puntos por objetivos 
no alcanzados, como el de la Figura 2. El juego se desarrolla por turnos, y en cada turno el jugador solo puede realizar una de las siguientes acciones:

1. Coger una o dos cartas de recurso para la construcción de la ruta, en base a una serie de reglas.

2. Cubrir un recorrido, y por tanto utilizar los recursos e incrementar su puntuación en el tablero (ver borde del tablero en la Figura 3).

3. Seleccionar nuevos destinos, aumentar el número de objetivos, compromisos sobre el alcance del proyecto en base a un conjunto de reglas, teniendo en cuenta el riesgo de no alcanzar dicho objetivo, y por tanto la pérdida de puntos que dicha mala estimación supondría.

El desarrollo del juego implica que los competidores se pueden estorbar en la construcción de las rutas, véase la figura 3, donde los equipos naranja y rojo han construido algunos recorridos. En la Figura 3 se muestran los recorridos ocupados por cada equipo, y en negro los recorridos por cubrir. Si, por ejemplo, el equipo naranja tuviera como objetivo completar la tarea o recorrido para conectar ambas ubicaciones, según la carta de la Figura 2, ahora no podría ir conectando Granada y Málaga, sino que debería de eliminar esa tarea y la correspondiente a Málaga-Cádiz, suponiendo que esta fuera su planificación. Y, definir nuevas tareas, por ejemplo: Córdoba-Sevilla con Sevilla-Cádiz. Por tanto, hay modificaciones en el alcance estimado de los objetivos. El juego impone que los participantes se adapten a los cambios. Por último, el juego finaliza con el primer equipo que consume las unidades construibles que tiene asignadas, en ese momento, se suman los puntos alcanzados por los recorridos realizados, los objetivos cumplidos (ubicaciones conectadas en el grafo), los puntos asociados al equipo que construye la ruta más larga, y finalmente se detraen los puntos por los objetivos no alcanzados, compromisos del proyecto no cumplidos o abordados. Hay que indicar que esto implica que el equipo que cierra la partida, el proyecto, no tiene por qué ser el ganador.

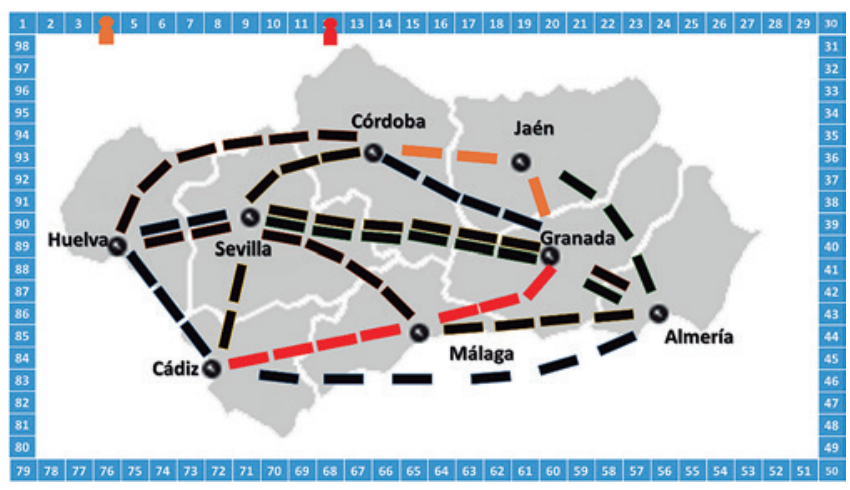

Figura 3. Ejemplo de situación de avance de un tablero.

\section{Metodología ágil y aplicación propuesta}

La misión del juego es alcanzar el número máximo de puntos, $y$, por tanto, ser la empresa con mayor beneficio en el desarrollo de la carrera de construcción de rutas logísticas. El desarrollo del proyecto requiere de cambios en el alcance del proyecto que dependerán del comportamiento de la competencia, por lo que siguiendo el manifiesto ágil (Beck, K.M., Beedle, M., Bennekum, A.V., Cockburn, A., Cunningham, W., Fowler, M., Grenning, J., Highsmith, J., Hunt, A., Jeffries, R., Kern, J., Marick, B., Martin,
R.C., Mellor, S.J., Schwaber, K., Sutherland, J., \& Thomas, 2001), en el desarrollo del juego priman las interacciones entre los participantes, la construcción real de los recorridos, la respuesta a los cambios, debida a que la competencia pueda adelantarse y escoger caminos que se habían planificado, como en el caso explicado con anterioridad. Esto supone que existe una cierta dificultad a la hora de seguir el plan, y, por lo tanto, el juego requiere de la adaptación de las propuestas de desarrollo.

Alcanzar una ruta, satisfacer al cliente, no supone que seamos más rápidos y eficientes, puesto que hay que adaptarse al cambio, si bien, no finalizar un destino está penalizado en el juego. Por otra parte, un cambio en la conexión de rutas, podría suponer una ventaja competitiva en futuros turnos de la partida, ya que podemos reconvertir un camino más largo en una oportunidad al escoger nuevos destinos más adelante.

\section{Aplicación del método SCRUM}

La idea de "Lean" es maximizar el valor para el cliente mientras se reduce el desperdicio, esta idea orienta la metodología SCRUM para proyectos donde la visión ágil se centra en agregar valor al negocio minimizando las tareas. Para ganar en el juego se requiere que el valor alcanzado sea el máximo, y los objetivos no alcanzados, los riesgos comprometidos en el proyecto, sean los mínimos por lo que la metodología ágil es ideal para la generación de valor en el juego.

El juego de mesa tiene una dependencia del azar, si queremos saber qué hacer, la respuesta es desconocida hasta obtener las cartas, donde responder a, ¿cómo?, es predecible. Entendemos que el alumno no tiene experiencia previa, por lo que se basa en "probar, detectar y responder" (Stacey, 2007).

La medida del progreso la tenemos en el número de tramos construidos, si bien su valor se encuentra en el tamaño del tramo y se ve reflejado en una tabla de puntos asociados a la longitud del tramo. La línea base del alcance del proyecto se encuentra en el número de partes de un recorrido que nos quedan, y el valor actual del proyecto se ve reflejado en los puntos conseguidos en los recorridos construidos, que se identifican en el entorno del tablero (Figura 4) y que nos indica cómo vamos respecto a nuestros competidores.

El desarrollo del proyecto no se puede sobrecargar en tareas, ni en objetivos, puesto que abordar más alcance del posible sería penalizado en el final del desarrollo del juego, si el objetivo no ha sido satisfecho.

La metodología ágil basada en SCRUM, sigue el siguiente diagrama de la Figura 4, donde los sprints, tienen como objetivo la entrega de valor, en este juego, puesto que se van a ir generando recorridos que serán propiedad de la empresa. Si bien el desarrollo del juego puede ser abordado desde varias perspectivas, se incita al alumno a un desarrollo ágil, por ser la estrategia incremental (no se pretenden hacer todos los destinos desde el principio) en la asunción de riesgos, y aplicar el alcance de forma iterativa (se adquieren nuevos destinos cuando se han alcanzado los anteriores).

En el juego, se refleja la aplicación del ciclo de Deming, donde:

1. Plan (P, planificar): se deciden los objetivos (tarjetas de destino) y la estrategia en la construcción de rutas (conjunto de recorridos).

2. Do (D, hacer): Hay tres posibilidades de acción en el juego, por orden de prioridad, se puede usar el turno:

- En primer lugar, para construir un recorrido antes de que se adelante otro jugador, es decir, obtener valor en el proyecto. 


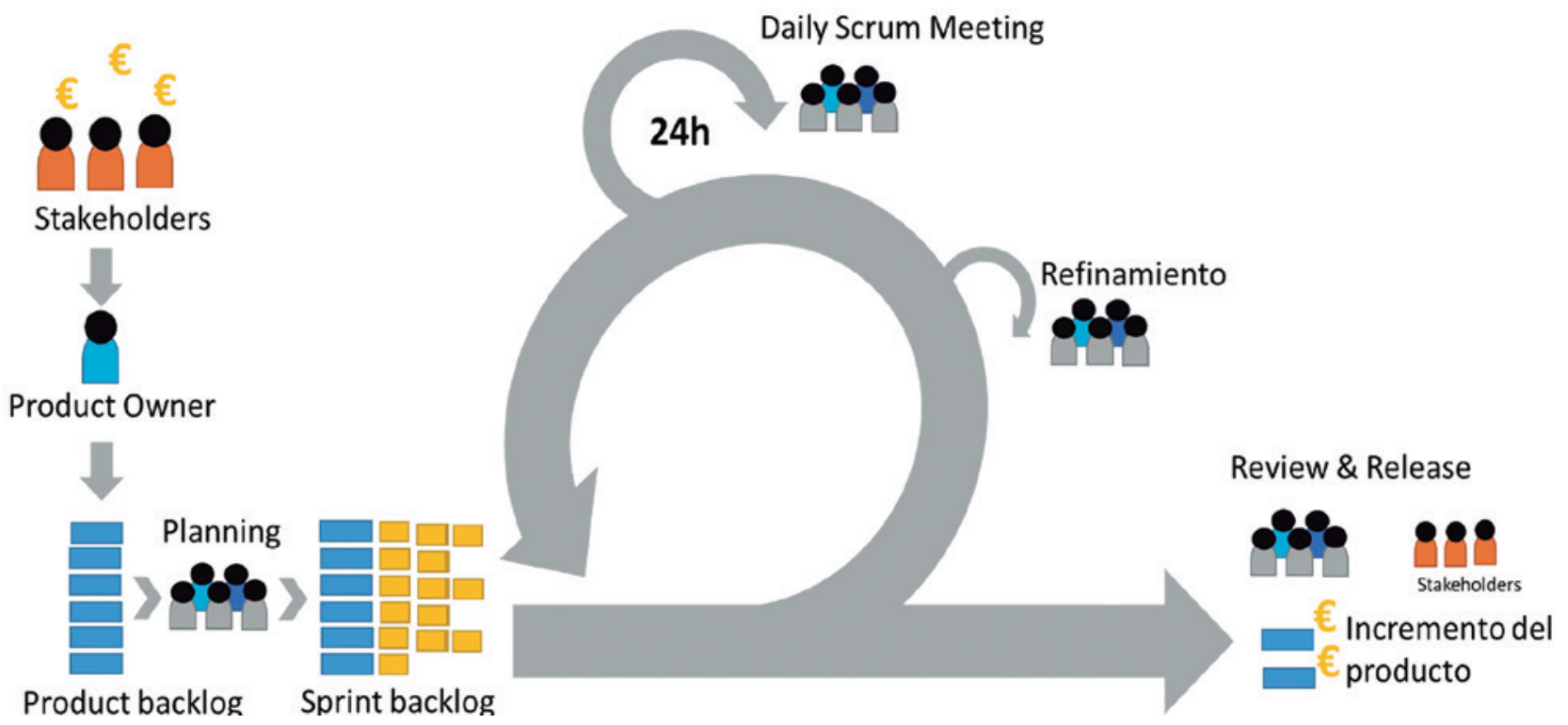

Figura 4. El proceso en SCRUM.

- En segundo lugar, para recoger recursos, con los que poder avanzar en la construcción, eliminación de tareas pendientes del proyecto.

- En tercer lugar, obtener nuevos objetivos o rutas, ampliar tareas/objetivos de alcance.

3. Check (C, verificar): durante el resto del turno, los contrincantes actúan, por lo que se puede verificar el resultado de la planificación.

4. Act (A, actuar): se realizan acciones correctoras al plan de rutas, se priorizan acciones, se identifican recorridos alternativos (se modifican las tareas), etc.

\subsection{Identificando conceptos: Roles, Artefactos y Eventos.}

En la metodología SCRUM (Keith, 2010), los roles, responden a la pregunta, ¿Quiénes participan en el proyecto?. En el juego se pueden detectar esos componentes: las partes interesadas o el cliente (Stakeholders), se encuentran representados en la baraja de cartas de ubicaciones a conectar, dado que esta baraja representa los objetivos que se quieren alcanzar. El equipo de trabajo (Scrum Team), los participantes en el desarrollo del proyecto serán los alumnos encargados de la elección de recursos de la baraja y la construcción de recorridos en el juego. El dueño del producto (Product owner), será el alumno encargado de la elección de las cartas de destino en cada sprint (pero no de la construcción de recorridos), y del seguimiento de resultados que se realizará con un Excel donde el alumno irá mostrando el estado del proyecto. Como veremos más adelante, en la figura 4, este rol se encargará del apoyo en la construcción de la pila del producto y de participar en algunas reuniones. Por último, el Scrum Master, ayudará en la evaluación de los objetivos, eliminación de impedimentos en el equipo de trabajo y reuniones.

En Scrum, los artefactos, responden a la pregunta, ¿Sobre qué se trabaja?, en el juego se pueden detectar estos tres componentes: La pila del producto (Product backlog), representa el desarrollo o alcance del proyecto, en el juego se construye a partir de la elección de cartas, donde los requisitos son presentados mediante una descripción técnica (relaciones de destinos que deben ser conectados). Y que se pueden presentar mediante el uso de historias de usuario (Beas, 2017). La lista de destinos comprometidos por cada equipo, supone la definición de una lista priorizada de requisitos, requiere de la presentación de las tareas y los órdenes de prioridad. En el desarrollo del backlog (Sutherland, 2013) o las tareas que hay que desarrollar en el proyecto, la simplicidad de la solución es primordial, siguiendo la idea de que en el desarrollo de un sprint "menos es más", debemos asegurar que los objetivos (características o tareas del cliente) más valiosos serán entregados primero. En el juego debemos enfocarnos en el problema real, si bien la planificación puede fallar por retrasarnos frente a competidores, se requiere consensuar en los equipos el comportamiento o estrategia más efectiva.

La pila del Sprint (Sprint Backlog), representan las tareas, recorridos o ítems que se quiere desarrollar durante el Sprint. Dado un destino, se identifican los caminos de la ruta, es decir, el valor incremental de la ruta (dado que representan puntos en el juego). Por último, en el Excel se muestran los objetivos alcanzados, el incremento del producto o estado actual del desarrollo del proyecto.

En Scrum, los eventos, son las situaciones de interacción y coordinación del proyecto, y también quedan reflejados en el juego. La planificación (Planing), se realiza tras desarrollar el Product Backlog (Figura 4), en este evento se debe definir el trabajo a realizar por el equipo en el sprint, para lo cual se debe valorar la velocidad y capacidad del proyecto, y se construye el Sprint bac$\mathrm{klog}$, lista de compromisos o requisitos que se quieren alcanzar del Product Backlog. El refinamiento de la pila, gestiona el mantenimiento de la propuesta, ya que la competencia puede realizar recorridos que teníamos planificados y habría que reorganizarse.

La reunión diaria (daily meeting), una reunión rápida que respondería en el juego a ¿qué hice en la última tirada (día de trabajo)?, ¿qué voy a hacer en esta tirada?, y, por último, ¿existe algún impedimento o problema?

Finalmente, quedan dos eventos, la revisión del sprint (Sprint Review) y la retrospectiva (Retrospective), que serían las reuniones al final de Sprint, donde los participantes, puedes revisar los indicadores, el valor alcanzado, y ver que se puede mejorar en la estrategia, analizar esta y comentar las acciones a tomar. 


\section{Conclusiones}

La experiencia fue aplicada en un grupo de alumnos de forma experimental y la opinión general fue satisfactoria. El juego permite incorporar y explicar todos los conceptos que componen la metodología ágil, por lo que se trata de un enfoque de aprendizaje activo para aprender los principios de desarrollo ágil y en especial el método Scrum. El juego aporta dinamismo, motivación y una aplicación integral de la metodología y variación de las tareas durante la ejecución del juego.

\section{Referencias}

Beas, J. M. (2017). Historias de Usuario. http://jmbeas.es/guias/historias-de-usuario/

Beck, K.M., Beedle, M., Bennekum, A.V., Cockburn, A., Cunningham, W., Fowler, M., Grenning, J., Highsmith, J., Hunt, A., Jeffries, R., Kern, J., Marick, B., Martin, R.C., Mellor, S.J., Schwaber, K., Sutherland, J., \& Thomas, D. (2001). The Agile Manifesto. Agile ALLIANCE, 6(1). http://www.agilemanifesto. org

Castán, A. R., Ruano, J. A. P., Municio, Á. M. G., \& Virginia San José Rodríguez, D. (2019). Gamificación en Ingeniería de Organización: "Cubioska" un juego de Role en Entorno Web. Direccion y Organizacion, 69, 62-81. https://doi.org/10.37610/ dyo.v0i69.561
Escudero-Santana, A., Aparicio-Ruiz, P., Grosso-de-la-Vega, R., \& Rodríguez-Palero, M. (2019). La colaboración Universidad-Empresa como elemento motivador. Un estudio de caso. Aula Abierta, 48(4), 435-446. https://doi.org/10.17811/ rifie.48.4.2019.435-446

Fernandes, J. M., \& Sousa, S. M. (2010). PlayScrum - A card game to learn the scrum agile method. 2nd International Conference on Games and Virtual Worlds for Serious Applications, VS-GAMES 2010, 52-59. https://doi.org/10.1109/VS-GAMES.2010.24

Keith, C. (2010). Agile Game Development With Scrum (A. Wesley (ed.)).

Petri, G., von Wangenheim, C. G., Hauck, J. C. R., \& Borgatto, A. F. (2019). Effectiveness of games in software project management education: An experimental study. Journal of Universal Computer Science, 25(7), 840-864. https://doi.org/10.3217/jucs025-07-0840

Sibona, C., Pourreza, S., \& Hill, S. (2018). Origami: An active learning exercise for scrum project management. Journal of Information Systems Education, 29(2), 105-116. http://jise.org/ Volume29/n2/JISEv29n2p105.html

Stacey, R. D. (2007). Strategic management and organisational dynamics: The challenge of complexity to ways of thinking about organisations. Pearson education.

Sutherland, K. S. y J. (2013). La Guía de Scrum. https://www.scrumguides.org/docs/scrumguide/v1/scrum-guide-es.pdf 
